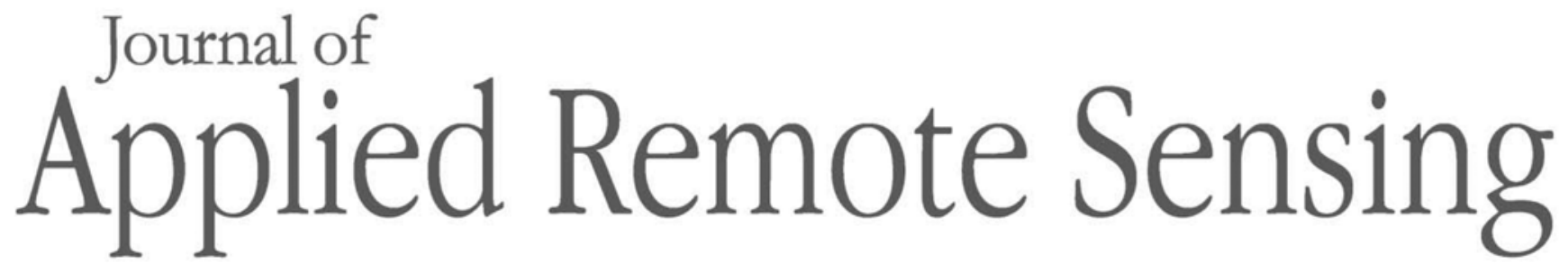

RemoteSensing.SPIEDigitalLibrary.org

\title{
Accurate sea-land segmentation using ratio of average constrained graph cut for polarimetric synthetic aperture radar data
}

Xiaoqiang She

Xiaolan Qiu

Bin Lei 


\title{
Accurate sea-land segmentation using ratio of average constrained graph cut for polarimetric synthetic aperture radar data
}

\author{
Xiaoqiang She, ${ }^{\mathrm{a}, \mathrm{b}, \mathrm{c}, *}$ Xiaolan Qiu, ${ }^{\mathrm{a}, \mathrm{b}, \mathrm{c}}$ and Bin Lei ${ }^{\mathrm{a}, \mathrm{b}, \mathrm{c}}$ \\ ${ }^{a}$ Chinese Academy of Science, Key Laboratory of Technology in Geo-spatial Information \\ Processing and Application System, Institute of Electronics, Beijing, China \\ ${ }^{\mathrm{b}}$ Chinese Academy of Science, Institute of Electronics, Beijing, China \\ ${ }^{c}$ University of Chinese Academy of Science, Beijing, China
}

\begin{abstract}
Separating sea surface and land areas in synthetic aperture radar (SAR) images is challenging yet of great importance to coastline extraction and subsequent coastal classification. Results of the previous state-of-art methods often suffer from a number of limitations that arise from the presence of the speckle effect and the inadequate returned signal around the boundaries. We propose a graph cut (GC)-based approach to tackle these limitations and achieve accurate sea-land segmentation results. To be more specific, as the first step, three powerful multipolarization features are extracted from the polarimetric SAR data as descriptors to fully characterize the sea area and land area. Starting from that, seeds of the sea and land are selected automatically to build the prior model for GC. Based on the prior model, we construct the undirected graph in GC using the multipolarization descriptors. Finally, we incorporate the ratio of average operator to eliminate the speckle effect and get finer results for some finer structures. Experiments on Radarsat-2 quad-polarization images demonstrate significantly improved results of our proposed algorithms compared with several state-of-the-art methods in terms of both quantitative and visual performance. (C) The Authors. Published by SPIE under a Creative Commons Attribution 3.0 Unported License. Distribution or reproduction of this work in whole or in part requires full attribution of the original publication, including its DOI. [DOI: 10.1117/1.JRS.11.026023]
\end{abstract}

Keywords: polarimetric synthetic aperture radar; multipolarization feature; edge constraint; graph cut.

Paper 16910 received Dec. 1, 2016; accepted for publication May 9, 2017; published online May 27, 2017.

\section{Introduction}

Sea-land segmentation in synthetic aperture radar (SAR) images is an important step for several important tasks, such as coastline extraction, ship detection, and coastal monitoring. ${ }^{1-5}$ However, sea-land segmentation in SAR images is not as a simple work as in photographic images. In the latter one, thresholding methods, for instance the Otsu's method ${ }^{6}$ and the local adaptive threshold method (LATM), ${ }^{7}$ are frequently used and achieve satisfactory segmentation results. The difficulties brought by SAR images mainly lie in two aspects: one is poor sea-land discrimination, which may lead to discontinuous boundaries, the other is the presence of the speckle effect. ${ }^{1,2}$ The first one is that the retuned SAR signal from the rough sea surface may exceed or be equal to that from the nearby land area. The second one, which is generated by scatters that are smaller than the SAR resolution elements, makes the segmentation task more complicated. As a consequence, sea-land segmentation in SAR images is difficult even for experienced interpreters.

Several methods that aim to solve the problems in SAR images have been proposed in the last decade. ${ }^{1-3,5,8,9}$ Among them, in the era of the single SAR images, the commonly used sea-land

*Address all correspondence to: Xiaoqiang She, E-mail: sxq@mail.ustc.edu.cn 
segmentation methods can only exploit the magnitude information and cannot achieve ideal results. As a comparison, recent algorithms using polarimetric SAR (PolSAR) data are populated thanks to the successful launch of several PolSAR satellites. PolSAR offers a brand-new view for sea-land segmentation since it provides more features compared with single PolSAR. ${ }^{6,10}$ These multipolarization features that can represent different scattering mechanisms are extracted from scattering decomposition and eigenvalue analyses. ${ }^{11,12}$ The existing methods that utilize SAR polarization information mainly focus on simply using the multipolarization features by several typical image processing methods, such as gray-level thresholding, ${ }^{5,8}$ machine learning, ${ }^{9}$ and multipolarization feature-based segmentation. ${ }^{10,13}$ As a result, the limitations caused by ambiguous sea-land separation and the speckle effect are not fully tackled by current methods.

In addition, with the improvement of the spatial resolution of SAR sensors, more geomorphic information is presented in SAR images in detail, which makes the segmentation problem more challenging. For instance, the tidal creeks on the intertidal area may be considered as land while some elongate structures may be difficult to detect. In this case, correct description of the boundary details is critical to the segmentation accuracy. ${ }^{3}$

In this paper, we propose a graph cut (GC)-based approach integrating multipolarization features and edge information to address the sea-land segmentation problems for SAR images. GC is an efficient framework that can achieve satisfactory two-value segmentation by two steps: transferring an image to an undirected graph and finding a cut through the graph with minimal cumulative cost. ${ }^{3,10} \mathrm{GC}$ can find a globally optimal segmentation for an image and generate a good balance of local terms at the same time. In GC, the impact of the ambiguous boundaries can be reduced by the local term, which incorporates neighborhood information and ensures a continuous shoreline. ${ }^{14}$ However, traditional GC belongs to the category of semisupervised segmentation methods that require users to specify foreground and background seeds for prior modeling. ${ }^{15}$ In this paper, improvements have been made on the basis of the GC framework in the view of the PolSAR data to achieve sea-land segmentation accurately with automatic seed selection. Three powerful multipolarization features are extracted from the quad-PolSAR data, i.e., the total power span, the polarimetric entropy $H$, and the mean scatter angle $\alpha$. For each pixel in the PolSAR image, a vector is formed by the three features as its multipolarization descriptor. Automatic seed selection can hence be possible by locating the pixels in the $H-\alpha$ plane based on the scattering mechanism of the sea and land. The selected seeds enable us to build the prior models for GC. Problems raised from the speckle effect are addressed by introducing edge constraint when modeling the boundary item in GC. The edge constraint is extracted by an edge map that is generated from the four-channel PolSAR image by the ratio of average (ROA) algorithm, which is significant for improving its speckle noise depressing ability. ${ }^{16}$ Our work significantly differs with previous works in three aspects:

1. Reliable and sufficient seeds are selected in a completely automatic way based on the $H-\alpha$ plane and enable us to build the prior models for GC.

2. A multipolarization descriptor that fuses the total scatter power span, polarimetric entropy $H$, and mean scatter angle $\alpha$ is proposed to build the graph model in GC.

3. Edge map that is generated from the PolSAR images by the ROA algorithm is used as edge constraint for modeling the boundary term in GC. The edge constraint is helpful in both reducing the speckle effect and avoiding the under-segmentation for some thin and elongated structures.

\section{Methodology}

In this section, we propose a sea-land segmentation method for PolSAR images. The proposed method can be divided into three parts. First, the $H-\alpha$ decomposition ${ }^{11}$ is applied to extract multipolarization features. The latter is used both in seed selection and the forming of the multipolarization descriptor. Then, the edge map is generated from the four channels of quad-polarization images by the ROA algorithm. Finally, an edge-constrained GC was applied to separate sea and land. Figure 1 shows the flowchart of our method. 
She, Qiu, and Lei: Accurate sea-land segmentation using ratio of average constrained graph cut...

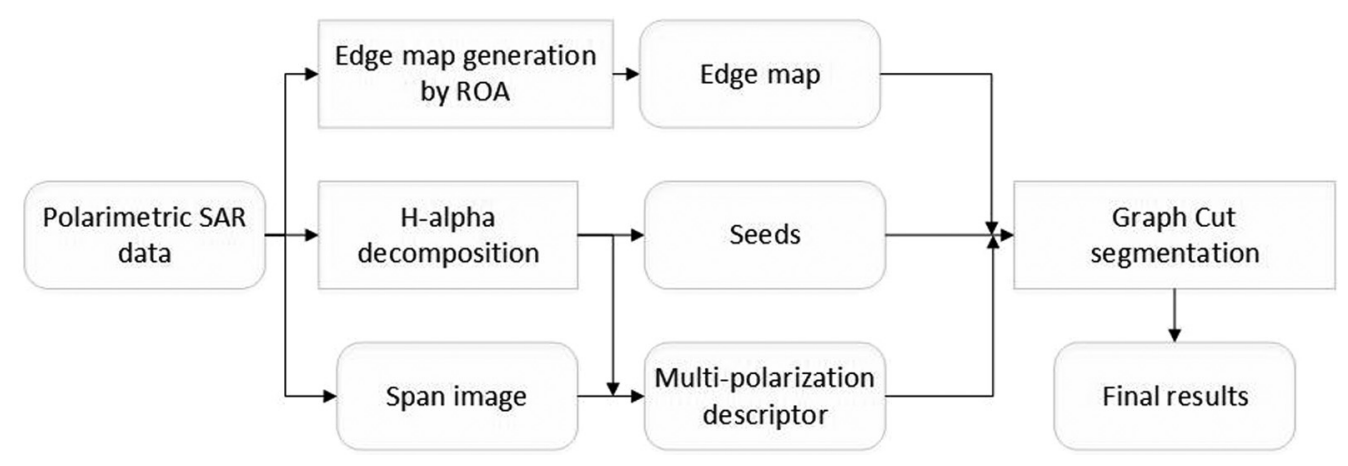

Fig. 1 Flowchart of the proposed segmentation method.

\subsection{Multipolarization Descriptor and Automatic Seed Selection}

PolSAR has attracted much attention since it can provide much more detailed information in comparison with single PolSAR in variant applications. In the sea-land segmentation case, we extract multipolarization features as descriptors to characterize the properties of the sea and the land. Based on the multipolarization descriptors, reliable seeds are selected to build the prior model for GC, and the undirected graph in GC is constructed subsequently.

\subsubsection{Synthetic aperture radar polarimetry features}

PolSAR data can offer a different view on describing the observed target surface by methods that exploit the combined information of the backscattering coefficients, among which the most famous one is the $H-\alpha$ decomposition. ${ }^{11}$ The definition of the $H-\alpha$ decomposition is based on the polarimetric coherency $T_{3}$ matrix

$$
T_{3}=\frac{1}{L} \sum_{n=1}^{L} k k^{* \mathrm{~T}}=\sum_{i=1}^{3} \lambda_{i} u_{i} u_{i}^{* \mathrm{~T}}
$$

where $L$ is the number of samples included in averaging and $k$ indicates the Pauli scattering

$$
k=\frac{1}{\sqrt{2}}\left[\begin{array}{lll}
S_{\mathrm{HH}}+S_{\mathrm{VV}} & S_{\mathrm{HH}}-S_{\mathrm{VV}} & 2 S_{\mathrm{HV}}
\end{array}\right]^{\mathrm{T}},
$$

where the elements $S_{\mathrm{HH}}$ and $S_{\mathrm{VV}}$ produce the power return in copolarized channels, and the element $S_{\mathrm{HV}}$ produces the power return in the cross-polarized channel. $\mathrm{H}$ and $\mathrm{V}$ represent the linear horizontal $(\mathrm{H})$ and vertical $(\mathrm{V})$ polarizations separately. The polarimetric entropy $H$ is derived from the eigenvalues $\lambda_{i}$ given by

$$
H=-\sum_{i=1}^{3} P_{i} \log P_{i}
$$

where $P_{i}$ is the pseudoprobability as

$$
P_{i}=\frac{\lambda_{i}}{\sum_{i=1}^{3} \lambda_{i}}
$$

The mean scatter angle $\alpha$ is computed from the eigenvectors

$$
\alpha=\sum_{i=1}^{3} P_{i} \cos ^{-1}\left[\left|u_{i}(1)\right|\right] .
$$

The polarimetric entropy is a measure of the randomness of the scattering processes and ranges from 0 to 1 . In a remote-sensing image, the borders between the sea and land can be seen as a unit 


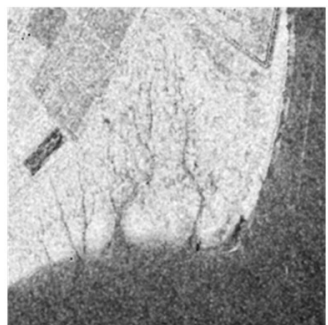

(a)

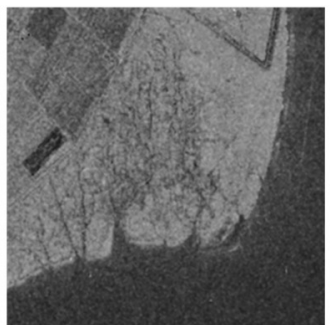

(b)

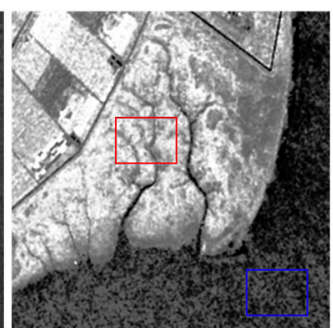

(c)

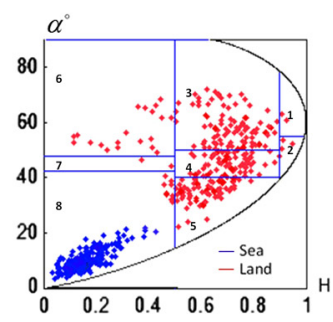

(d)

Fig. 2 Examples of the multipolarization features and the $H-\alpha$ plane. (a) Polarimetric entropy $H$. (b) Mean scatter angle $\alpha$. (c) Total power span. (d) The $H-\alpha$ plane, in which the red points demonstrate the locations of the land area marked as red box in (c), and the blue points indicate the location of the sea area marked as blue box in (c).

of the edge points, whereas the edge strength per unit area can be measured by the texture strength. The polarimetric entropy has been proved to be closely related to the polarizationdependent variation of texture due to it being sensitive to the scattering randomness. Therefore, the polarimetric entropy is helpful in measuring the differences of scattering randomness between the sea and land. The mean scatter angle $\alpha$ takes values between $0 \mathrm{deg}$ and $90 \mathrm{deg}$. The increase of $\alpha$ indicates three scattering mechanisms, namely surface scattering, volume scattering, and double bounce scattering, respectively, which could measure the differences of surface roughness between the sea and land. ${ }^{12}$

Another common multipolarization parameter is the span, which is the total power of the polarimetric channels. Span is a combination of the information of the four channels and can offer more details than them. ${ }^{11}$ The definition of span is given as

$$
\text { Span }=\left|S_{\mathrm{HH}}\right|^{2}+\left|S_{\mathrm{HV}}\right|^{2}+\left|S_{\mathrm{VH}}\right|^{2}+\left|S_{\mathrm{VV}}\right|^{2} .
$$

In the SAR images, we have found that the sea surfaces are dominated by the Bragg surface scattering, which can be measured by the mean scattering angle $\alpha$, and land areas are more complicated in texture and intensity distribution. To fully characterize the sea and land, these three powerful multipolarization features are normalized between 0 and 1 and set into a vector for every pixel in the SAR image as a descriptor. The polarimetric entropy is between 0 and 1 by definition. The mean scatter angle $\alpha$ is normalized by dividing by 90 . The normalization of the span includes two steps: clipping the span image $S$ to $0.1 *(S)$ followed by linear normalization to 0,1. Examples of the three multipolarization features are shown in Fig. 2, in which the polarimetric entropy $H$ is demonstrated in 2(a), the mean scatter angle $\alpha$ is shown in 2(b), and 2(c) gives the image of the total power span.

\subsubsection{Automatic seed selection by $H-\alpha$ plane}

We have extracted three powerful multipolarization features from the PolSAR data, i.e., the total scatter power span, polarimetric entropy $H$, and mean scatter angle $\alpha$. Based on polarimetric entropy and mean scatter angle, Cloude and Pottier ${ }^{13}$ proposed a two-dimensional $H-\alpha$ plane that can represent an all random scattering mechanism, given in Fig. 2(d). This plane is subdivided into eight basic zones to characterize different classes of land covers based on their scattering mechanisms.

In the sea-land segmentation case, the sea areas usually have both low entropy and scatter angle values for its smooth surface, whereas the values of the land parts are usually relatively high for its relative complicated texture distributions. As a consequence, seeds selection for the sea and the land will be possible by locating the pixels in the $H-\alpha$ plane based on its entropy and scatter angle. Figure 2(d) shows the locations in the $H-\alpha$ plane of some sample pixels. In Fig. 2(d), locations of the land area marked as red box in Fig. 2(c) are represented as red points, and the blue points indicate the locations of the sea area that are marked as blue box in Fig. 2(c). It is clearly seen that the sea area mainly fall into the category with entropy value less than 0.5 and $\alpha$ value less than 42.5 deg, i.e., zone 8 as marked in Fig. 2(d). ${ }^{11}$ To this end, the seeds 


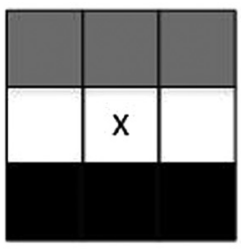

(a)

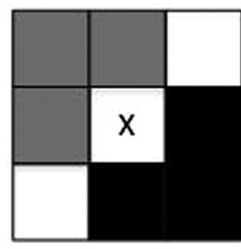

(b)

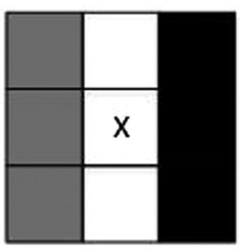

(c)

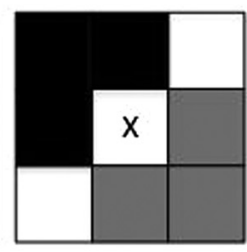

(d)

Fig. 3 Mask of ROA: target $A$ and target $B$ in the moving window for four typical directions. The directions are: (a) 0 deg, (b) $45 \mathrm{deg}$, (c) $90 \mathrm{deg}$, and (d) $135 \mathrm{deg}$.

selection work for the sea can be simplified by threshold works based on the $H-\alpha$ plane. In this paper, pixels that have $H$ values lower than 0.3 and $\alpha$ values lower than 30 deg are selected as seeds of the sea area. The seeds of the land are selected from the pixels with entropy values higher than 0.4 and with $\alpha$ values higher than $45 \mathrm{deg}$.

\subsection{Ratio of Average-Based Edge Map Generation}

The speckle appear in SAR images will lead the inaccuracy in sea-land segmentation work. Based on the multiplicative noise model, several studies have been developed to smooth the speckle noise. Among them, the ROA algorithm is often used in the present studies to address this problem in a variety of applications.

ROA is one of the most efficient edge detectors for SAR images, which belongs to the class of constant-false-alarm-rate operators and can be used to eliminate the speckle effect in SAR images by employing the ratio of neighboring pixels.

In ROA, a moving window is segmented to two subwindows $\mathrm{P}$ and $\mathrm{Q}$ along the assumed edge direction. Figure 3 shows the mask of ROA with four typical directions. ${ }^{13,17}$ For every direction, the ROA is expressed as

$$
R^{d}=\max \left(\bar{I}_{P}^{d} / \bar{I}_{Q}^{d}, \bar{I}_{Q}^{d} / \bar{I}_{P}^{d}\right),
$$

where the superscript $d$ indicates the direction, and $\bar{I}_{P}$ and $\bar{I}_{Q}$ denote the mean scattering intensities for $\mathrm{P}$ and $\mathrm{Q}$, respectively. The final result $R$ can be represented by the maximum ratio in all the directions in set $O$

$$
R=\max \left\{R^{d}, d \in O\right\} .
$$

In this paper, the ROA is applied to the four channels of quad-PolSAR images, i.e., $\mathrm{HH}, \mathrm{HV}, \mathrm{VH}$, and VV to extract their edge maps separately. The final edge map $R_{e}$ is given as the sum of the four edge maps in each pixel $i$

$$
R_{e}(i)=\sum_{m, n \in\{H, V\}} R_{m n}(i)
$$

In our test, we further normalized $R_{e}$ to $[0,1]$.

The resulting edge map $R_{e}$ can delineate the boundaries excluding the impact of speckle noise. Consequently, accurate sea-land segmentation will be possible by introducing the edge map into the GC framework.

\subsection{Edge Constrained Graph Cut}

We have proposed multipolarization descriptors to characterize the sea and the land by extracting features from the PolSAR data. In this end, seeds of the sea and land are selected automatically. Furthermore, edge map is generated by ROA depressing the speckle effect. Based on these, we accomplish the final segmentation work with our edge-constrained GC framework.

$\mathrm{GC}$ is an efficient two-class segmentation framework that is widely used in image segmentation works due to its compatibility and robustness. In GC, each pixel in an image is represented as a node in an undirected graph, and the image segmentation work is achieved by cutting 
through the graph with minimal cumulative cost, which is a typical energy minimization problem. The energy function for GC can be expressed as

$$
E(L)=\sum_{i \in I} D_{i}\left(L_{i}\right)+\lambda \sum_{(i, j) \in \xi} V_{i j}\left(L_{i}, L_{j}\right),
$$

where $L$ is the label map of the image $I$ for segmentation, $D_{i}(\cdot)$ is the data constraint, which corresponds to the likelihood that the node $i$ belongs to class $L_{i}$. Boundary item $V_{i j}$ measures the cost of assigning $i$ and $j$ with different labels and can ensure the continuity of the edges between the background and foreground. The positive constant $\lambda$ specifies the relative importance of the data constraint and the boundary item, which is set at 10 in our experiments. Set $\xi$ indicate the edges of all pairs of neighboring pixels. ${ }^{3}$ Based on the image $I$ and the edge set $\xi$, an undirected graph $G=\langle I, \xi\rangle$ can be built. In $G$, each node is connected to its neighboring pixels by so-called $n$-links and to the two terminal nodes by $t$-links. The $t$-links correspond to the global term and the $n$-links correspond to the boundary term in the energy function $E(L)$.

The most crucial step of constructing the graph is the assignment of link weights. In this step, we form a multipolarization feature space by the three features in the multipolarization descriptor, i.e., the total power span, the polarimetric entropy $H$, and the mean scatter angle $\alpha$. Prior models of the sea and land are built by the Gauss mixture models (GMMs) in the multipolarization feature space. In our experiments, the numbers of the components of the GMMs are 3 and 4 for the sea and land, respectively, because the land area usually has a relatively complicated distribution of texture. As a matter of fact, we find that the numbers of the components have little influence on the final segmentation results once they are selected in a reasonable range from 3 to 5 .

Based on the GMMs, the $t$-links of our graph model are defined as

$$
D_{i}\left(L_{i}\right)=-\log \left[p\left(\vec{x}_{i} \mid L_{i}\right)\right],
$$

where $p(\cdot)$ is one of the two GMM models that are determined by label $L_{i}$, and $\vec{x}_{i}$ indicates the three-channel multipolarization descriptor of the $i$ 'th pixel.

When calculating the $n$-links, we use the spatial distance between two multipolarization feature vectors and combine it with the edge maps that are generated from ROA, given by

$$
V_{i j}\left(L_{i}, L_{j}\right)=\exp \left[-\sigma_{i j} \cdot R_{i j} \cdot\left(\vec{x}_{i}-\vec{x}_{j}\right)^{2}\right] \cdot\left|L_{i}-L_{j}\right|,
$$

where $R_{i j}$ indicates the edge information between the $i$ 'th pixel and the $j$ 'th pixel, and the vectors $\vec{x}_{i}$ and $\vec{x}_{j}$ indicate the three-channel multipolarization descriptor of the $i$ 'th pixel and $j$ 'th pixel separately. The calculation of $R_{i j}$ is defined by the sum of the values of these pixels in edge map $R_{e}$

$$
R_{i j}=R_{e}(i)+R_{e}(j) .
$$

The coefficient $\sigma$ is calculated by

$$
\sigma_{i j}=\frac{1}{2\left\langle\left|\vec{x}_{i}-\vec{x}_{j}\right|^{2}\right\rangle}
$$

where $\langle\cdot\rangle$ indicates the integral average value.

Term $R_{i j}$ is important in two aspects: one is smoothing the speckle noise around the boundaries and the other is detecting some thin and elongated structures.

\section{Experiments}

In the experiment, the proposed method was evaluated and validated on the Radarsat-2 C-band SAR data. The processed images are selected from Radarsat-2 quad-polarimetric fine-resolution SAR images over Shanghai and San Francisco as described in Fig. 4. In Fig. 4, (a) is selected from data over Shanghai with size $2000 \times 2000$ and others are selected from data over San 


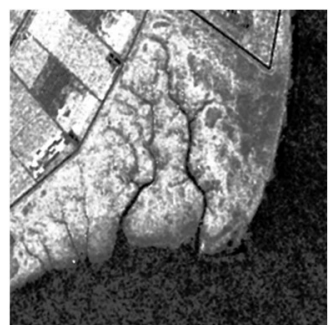

(a)

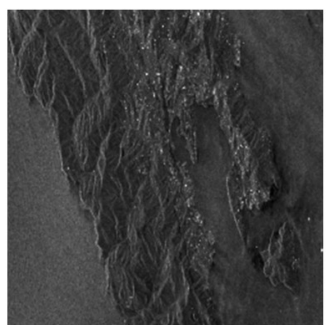

(b)

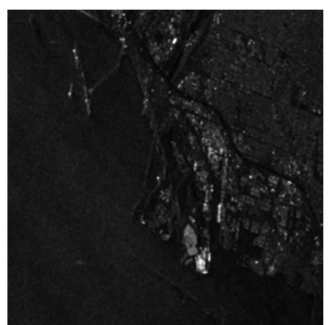

(c)

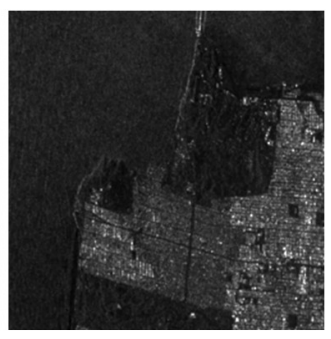

(d)

Fig. 4 Span images of the selected sea-land scenes: (a) intertidal area of Chongming Island, Shanghai, (b)-(d) different parts of San Francisco.

Francisco with size $2000 \times 2000,1000 \times 1000$, and $1000 \times 1000$, respectively. The ground truth images are labeled by our interpreters carefully to evaluate the experimental results. Comparative experiments are carried out based on subimages that are selected from the processed images including scenes of complex distribution of texture and complicated structures.

\subsection{Experimental Results}

Figure 5 shows the intermediate processing and segmentation results of the four selected images in Fig. 4. Figure 5(a) shows the span images the same as those in Fig. 4. Figure 5(b) shows the selected seeds for the land and sea that are displayed in red and blue, respectively. The seeds selection results can efficiently mark the sea and land. Edge maps that are generated from the ROA algorithm are shown in Fig. 5(c), in which the edges are delineated clearly. Figure 5(d)
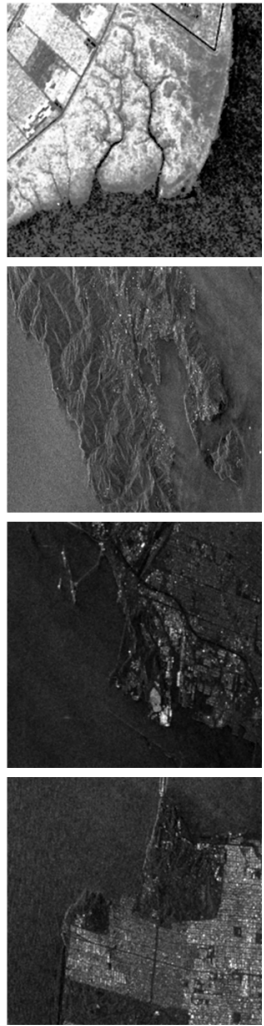

(a)
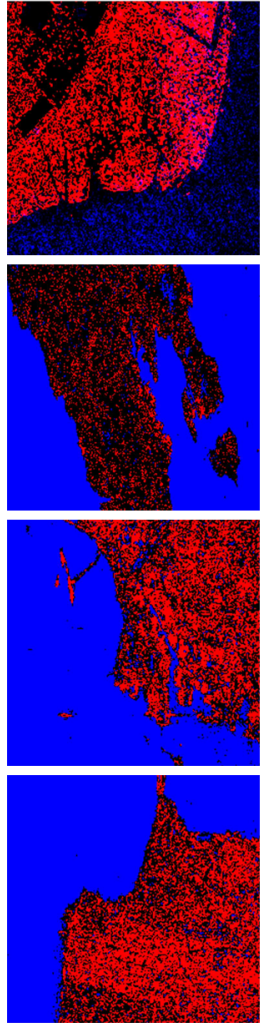

(b)
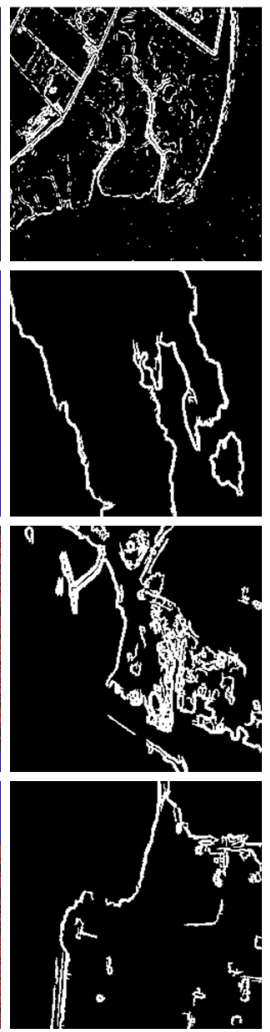

(c)
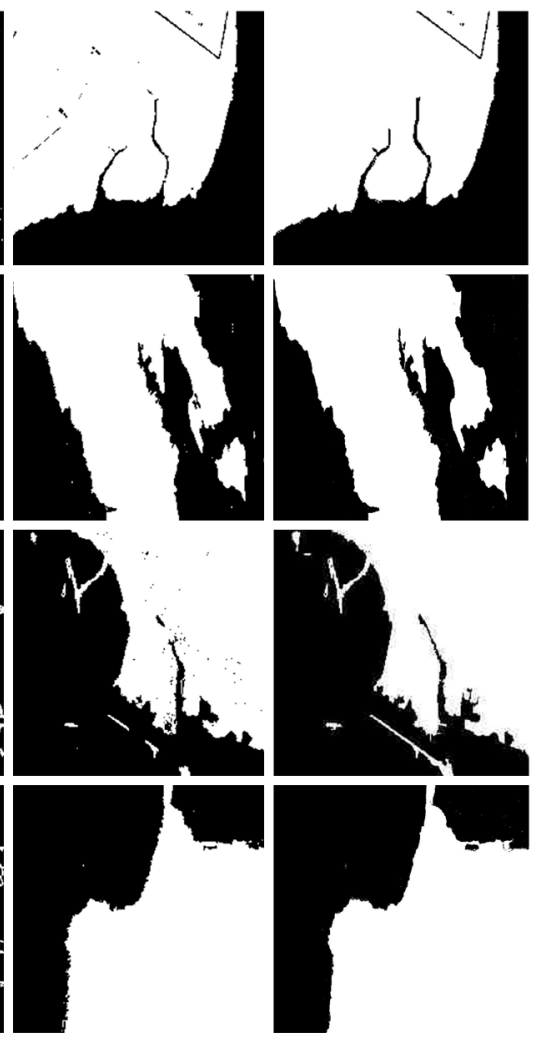

(d)

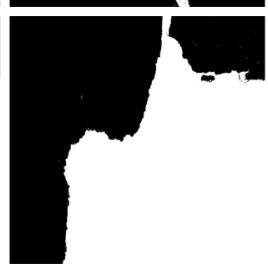

(e)

Fig. 5 Visual illustration of the multipolarization features, seed selection results, and edge maps of the four scenes in Fig. 3: (a) the span image, (b) selected seeds in which the seeds of the sea and land are displayed in blue and red respective while the black ones are unlabeled, (c) edge maps that generated by ROA, (d) segmentation results, and (e) ground truth that manually outlined. 
shows the final segmentation results, which can ensure continuous shorelines. In Fig. 5, the tidal creeks are detected accurately as described in the first row, and some slender structures are classified correctly as described in the third row. Figure 5 shows that the final results of our method can offer good visual matches compared with the ground truth that are given in Fig. 5(e).

\subsection{Quantitative and Visual Comparison}

We have demonstrated the effectiveness of the proposed method as applied to the Radarsat-2 PolSAR data. To be more complete, comparative experiments are carried out based on three baselines, i.e., $\mathrm{GC}^{15}$ without edge constraint and multipolarization descriptor, Hansch's algorithm, ${ }^{10}$ and LATM. $^{7}$ Among these three methods, GC is the basic framework of the proposed method, Hansch's algorithm is an extension of the GC framework for PolSAR data, and LATM is a typical thresholding method. In the comparative experiments, the traditional GC and Hansch's method use the same seeds used by our method to do the segmentation work. The building of an undirected graph is similar to the proposed method while the differences are that the multipolarization feature vector is replaced by the simple span image in building the GC model and the $n$-link weights are calculated without the edge constraints. For the LATM, the inputs are the span images. In the experiments of the Hansch's method, we use the same multipolarization feature vector as our method to build the $t$-links and use the complex Wishart distribution to build the $n$-links. In the experiments of traditional GC and Hansch's method, the $\lambda$ is set to 10 and the number of the components in the GMMs is set to 3 and 4 indicates the sea and land separately. We select subimages from the four testing images with complex shorelines and complicated structures. Visual illustrations of the segmentation results of different methods are presented in Fig. 6.
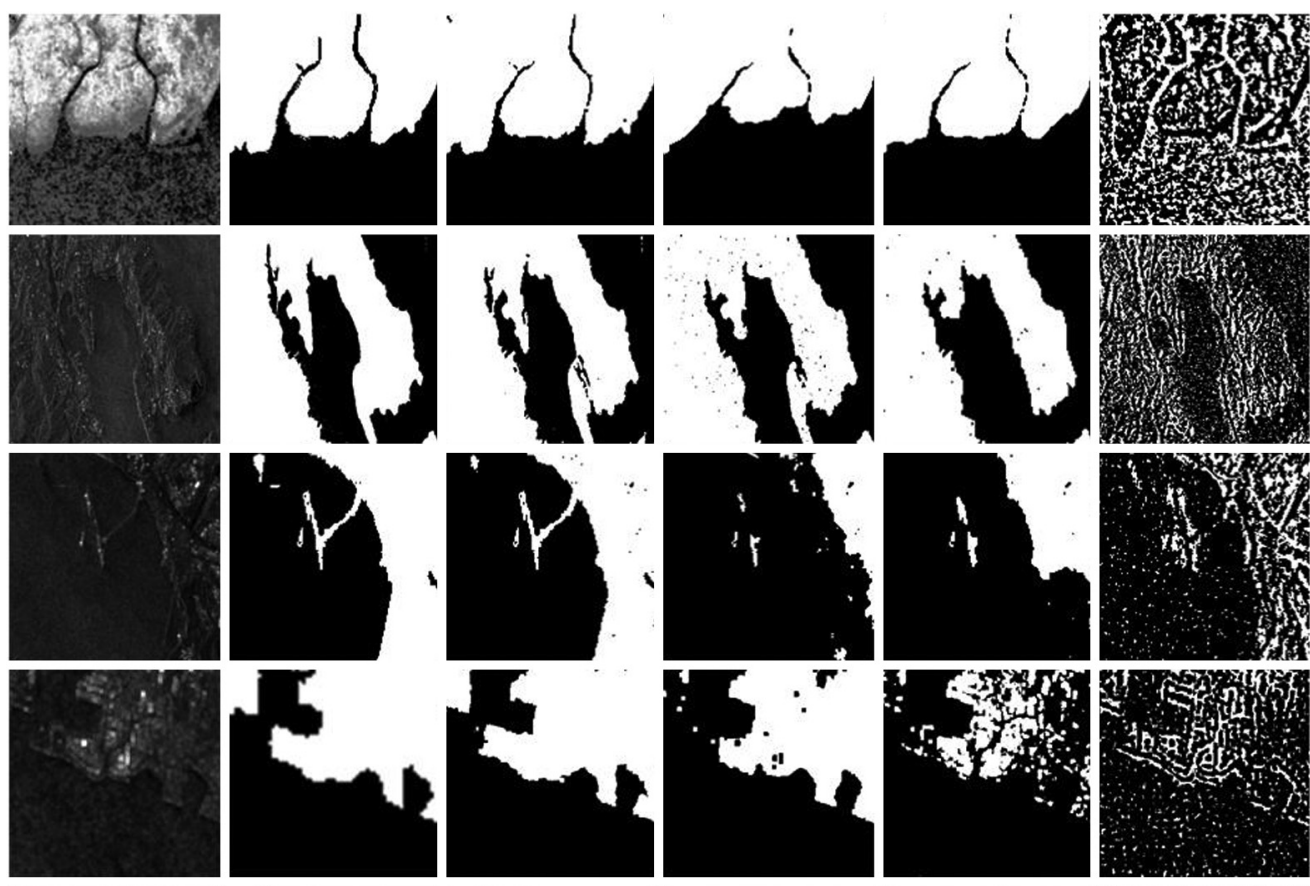

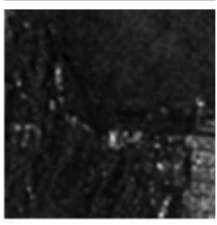

(a)

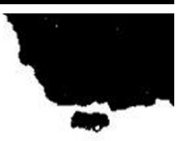

(b)

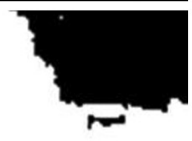

(c)

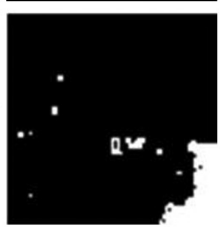

(d)
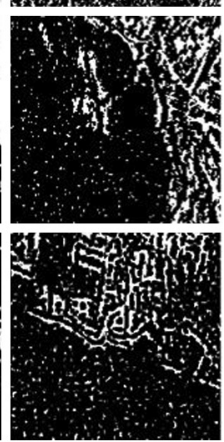

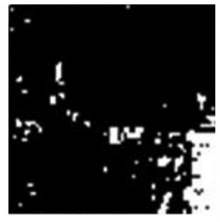

(e)

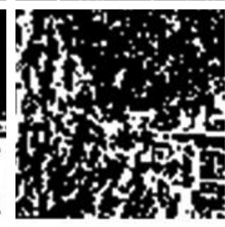

(f)

Fig. 6 Visual comparison of segmentation results of different methods. (a) Span image, (b) ground truth, (c) ours, (d) GC, (e) method by Hansch, and (f) LATM. 
Table 1 Average ROL, POL, ROS, and POS and their STD obtained with different methods. The STD indicates the standard deviation and the bold values indicate the best.

\begin{tabular}{lcccc}
\hline \hline Method & ROL \pm STD (\%) & POL \pm STD $(\%)$ & ROS \pm STD (\%) & POS \pm STD (\%) \\
\hline LATM & $32.58 \pm 2.91$ & $67.64 \pm 6.63$ & $79.24 \pm 14.14$ & $51.18 \pm 15.92$ \\
Method by Hansch & $82.88 \pm 18.49$ & $98.15 \pm 1.27$ & $98.02 \pm 1.24$ & $86.23 \pm 13.81$ \\
GC & $71.49 \pm 28.73$ & $98.34 \pm 1.85$ & $97.94 \pm 2.25$ & $82.35 \pm 13.54$ \\
Ours & $\mathbf{9 8 . 3 1} \pm \mathbf{1 . 3 0}$ & $\mathbf{9 8 . 3 5} \pm \mathbf{1 . 4 6}$ & $\mathbf{9 8 . 1 0} \pm \mathbf{1 . 6 9}$ & $\mathbf{9 8 . 2 8} \pm \mathbf{1 . 5 0}$ \\
\hline \hline
\end{tabular}

The results of the comparative experiment demonstrate that the results of our method are spatially consistent with few errors compared to the ground truth owing to the reliable seeds and speckle-reduced edge constraints. Specially, our method outperforms the comparative methods for the scenes that have thin and elongate structures, as presented in the first and third row in Fig. 6. Images in the second and fourth row in Fig. 6 demonstrate that our method performs well in the complex coastline case. The last row of Fig. 6 shows that detailed information can be extracted by our method accurately.

Quantitative analysis work is carried out by four measurement methods, i.e., recall of the land (ROL), precision of the land (POL), recall of the sea (ROS), and the precision of the sea (POS). ${ }^{3}$ We compute these measurements on the pixel level for the testing images in Fig. 6. In addition, we calculate the standard deviations (STD) of the four measurements for the testing images.

Table 1 presents the average ROL, POL, ROS, and POS that are calculated by the average of each image, and our method performs best on all the four measurements. Furthermore, the STDs, i.e., the STD of the testing images indicate that our method is the most stable one. Among the four measurements, the three comparative methods achieve relatively small ROL values owing to the ambiguous nature for sea-land separation in SAR images. ${ }^{1,2}$ In addition, POS values are small owing to the wind-roughed and speckled sea surface in SAR images, which may cause misclassification. All of these posed difficulties are caused by the presence of speckle and the returned signal from the roughed sea surface. By employing the ROA algorithm and multipolarization descriptor, our method addresses all these difficulties and achieves the best results.

\subsection{Effects of Introducing Edge Constraints}

The incorporation of the ROA-based edge map in our GC framework is aimed at reducing the speckle noise and avoiding under segmentation for some slender structures. To further validate the effectiveness, we conduct comparative experiments with the canny edge directed GC that is proposed in Dongcai Cheng's paper. ${ }^{3}$ In Cheng's work, the edge constraint in GC is the edge map that is generated by canny detector rather than ROA. The comparative results are shown in Fig. 7, in which (a) indicates the span images, (b) represents the canny edges, (c) shows the
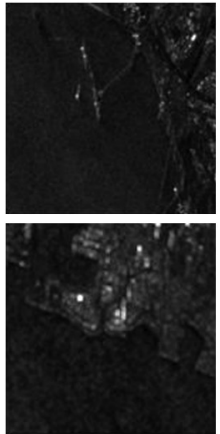

(a)

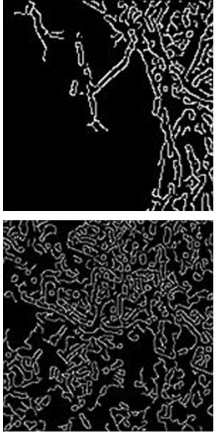

(b)

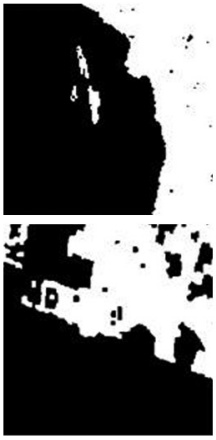

(c)

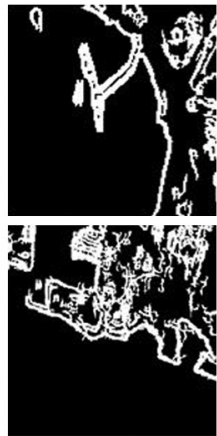

(d)

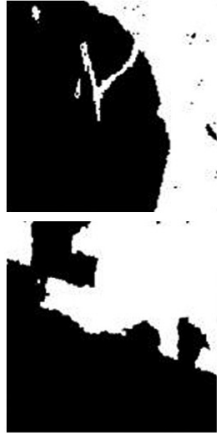

(e)

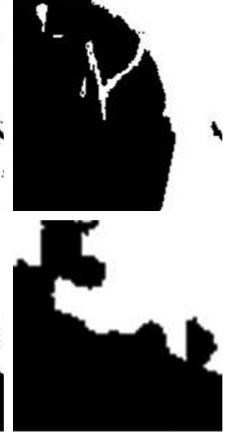

(f)

Fig. 7 Comparative experiments with canny edges constrained GC: (a) span images, (b) canny edges, (c) segmentation results of GC with canny edge constraints, (d) our ROA-based edge maps (e) segmentation results of ours, and (f) ground truths. 

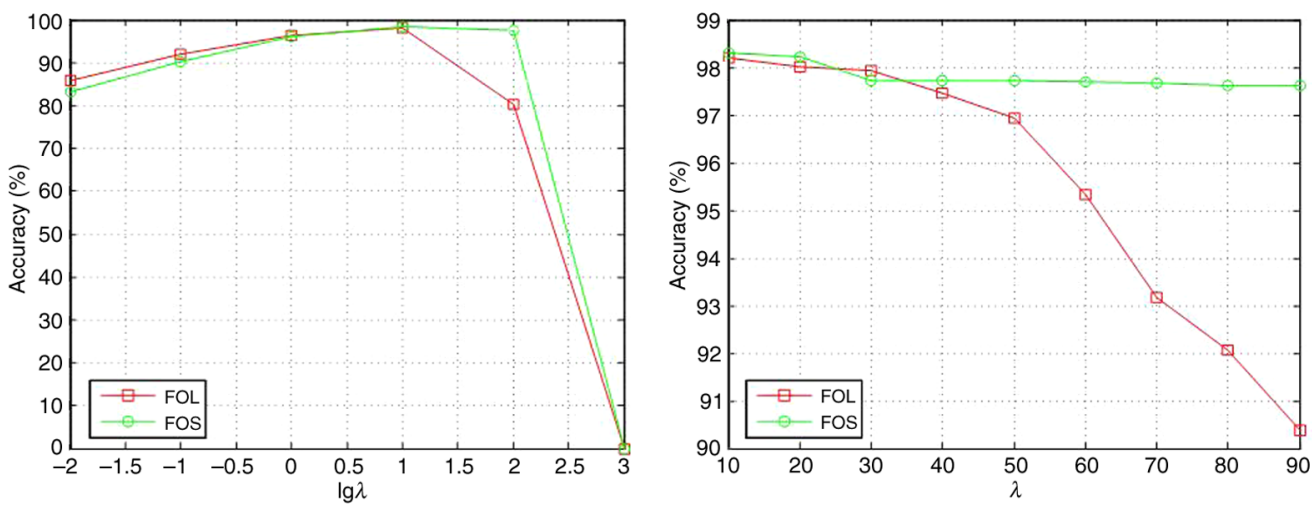

Fig. 8 Influence of value of $\lambda$.

results of canny edge directed GC, and the next two figures demonstrate the edge maps and segmentation results of the proposed method. Figure 7(f) shows the ground truths.

Experimental results show that our method is effective in some cases such as the thin structures, which may be easily corrupted by the speckle noise in SAR images, as presented in the first row of Fig. 7. The second row of Fig. 7 shows the results of a subimage with complicated distribution of texture and inadequate boundaries. The latter case may be caused by the signal returned from the water area that shares similar intensity compared with the nearby land as well as the speckle effect. In this case, our method outperforms the canny edge directed GC and achieves satisfactory results.

\subsection{Influence of Parameters}

\subsubsection{Selection of $\lambda$ in graph cut}

The parameter $\lambda$ balances the relative importance of the $n$-links and $t$-links in GC. When $\lambda$ is small, the segmentation results are dominated by the $t$-link weights, i.e., the global information and may lead to discontinuous boundaries. However, when the value of $\lambda$ is too large, the image will be over-smoothed and cause under-segmentation around the boundaries. In both cases, the accuracy of the sea-land segmentation will be decreased.

To validate the influence of the value of $\lambda$, this paper sets different values of $\lambda$ and compute the average ROL, ROS, POL, and POS on the testing images. Specially, the $F 1$-measure is used as the balanced performance measurement, ${ }^{18}$ given by

$$
\begin{aligned}
& \mathrm{FOL}=\frac{2 \mathrm{ROL} \cdot \mathrm{POL}}{\mathrm{ROL}+\mathrm{POL}}, \\
& \mathrm{FOS}=\frac{2 \mathrm{ROS} \cdot \mathrm{POS}}{\mathrm{ROS}+\mathrm{POS}},
\end{aligned}
$$

where FOL is the $F 1$-measure of the land and FOS is the $F 1$-measure of the sea. The experiments are divided into two parts: first, the value of $\lambda$ is set to a wide range from 0.01 to 1000 , and the results are shown in the left figure of Fig. 8; second, the value of $\lambda$ is chosen in a finer range from 10 to 90 as represented in the right figure of Fig. 8. The combination of both figures in Fig. 8 validates that the accuracy reach the best when the value of $\lambda$ is set to 10 .

\subsubsection{Influence of the number of components in the Gauss mixture models}

In our method, the GMMs are used to fit the distribution of the selected seeds of the sea and land. To study the influence of the number of components in the GMMs, we set different pairs of (sea, land) components in a range from 2 to 5 and compute the average ROL, ROS, POL, and POS on the testing images. As the land area usually has a relatively complicated distribution 
Table 2 Influence of the number of the components in the GMMs.

\begin{tabular}{lcccc}
\hline $\begin{array}{l}\text { Number of components in } \\
\text { the GMMs (sea, land) }\end{array}$ & ROL (\%) & POL (\%) & ROS (\%) & POS (\%) \\
\hline$(2,3)$ & 97.66 & 97.47 & 97.44 & 97.23 \\
$(2,4)$ & 97.79 & 98.22 & 98.11 & 97.68 \\
$(2,5)$ & 98.25 & 98.28 & 98.64 & 97.98 \\
$(3,4)$ & 98.31 & 98.35 & 98.10 & 98.26 \\
$(3,5)$ & 98.29 & 98.37 & 98.08 & 98.25 \\
$(4,5)$ & 98.31 & 98.70 & 98.09 & 98.14 \\
\hline \hline
\end{tabular}

of intensity, we set a larger number of the components in the GMMs for the land area. The results are represented in Table 2, from which it can be seen the numbers of the components have little influence on the final segmentation results once they are selected in a reasonable range from 3 to 5 .

\section{Conclusion}

A GC-based approach is proposed in this paper to address the sea-land segmentation problems for PolSAR images. In our work, the ambiguous sea-land separation problem is addressed by exploiting the neighborhood information in GC. Specially, we employ the multipolarization features to select seeds for the sea and land automatically and build a graph model for GC. The accurate segmentation result is achieved by utilizing the ROA-based edge constraints, which can address problems associated with the speckle effect around the boundaries as well as the under-segmentation for some finer structures. We conduct experiments on two sets of Radarsat-2 PolSAR data gathered over Shanghai and San Francisco and show our algorithm outperforms three traditional methods with nontrivial margins, which demonstrates the effectiveness of our method.

Further improvements in accuracy for the purpose of geographic mapping and land use classification would require more powerful multipolarization feature and additional modifications in the GC framework.

\section{References}

1. J. S. Lee and I. Jurkevich, "Coastline detection and tracing in SAR images," IEEE Trans. Geosci. Remote Sens. 28(4), 662-668 (1990).

2. D. C. Mason and I. J. Davenport, "Accurate and efficient determination of the shoreline in ERS-1 SAR images," IEEE Trans. Geosci. Remote Sens. 34(5), 1243-1253 (1996).

3. D. Cheng et al., "Efficient sealand segmentation using seeds learning and edge directed graph cut," Neurocomputing 207, 36-47 (2016).

4. W. M. Moon et al., "Radarsat-2 and coastal applications: Surface wind, waterline, and intertidal flat roughness," Proc. IEEE 98(5), 800-815 (2010).

5. A. Buono et al., "A multipolarization analysis of coastline extraction using x-band cosmoskymed SAR data," IEEE J. Sel. Top. Appl. Earth Obs. Remote Sens. 7(99), 2811-2820 (2014).

6. Y. Shu et al., "Dark-spot detection from SAR intensity imagery with spatial density thresholding for oil-spill monitoring," Remote Sens. Environ. 114(9), 2026-2035 (2010).

7. A. J. Burghardt, G. J. Kazakia, and S. Majumdar, "A local adaptive threshold strategy for high resolution peripheral quantitative computed tomography of trabecular bone," Ann. Biomed. Eng. 35(10), 1678-1686 (2007).

8. Y. Yu, "Automated delineation of coastline from polarimetric SAR imagery," Int. J. Remote Sens. 25(17), 3423-3438 (2004). 
9. X. F. Zhang, Y. H. Zhang, and W. F. Zhong, "Delineation of sea island using polarimetric SAR image," Remote Sens. Inf. 27(3), 34-39(2012).

10. R. Hansch, O. Hellwich, and X. Wang, "Graph-cut segmentation of polarimetric SAR images," in IEEE Int. Geoscience Remote Sensing Symp. (IGARSS), 1733-1736 (2014).

11. J. S. Lee and E. Pottier, Polarimetric Radar Imaging, CRC Press, Boca Raton, Florida (2015).

12. S. R. Cloude and E. Pottier, "A review of target decomposition theorems in radar polarimetry," IEEE Trans. Geosci. Remote Sens. 34(2), 498-518 (1996).

13. S. R. Cloude and E. Pottier, "An entropy based classification scheme for land applications of polarimetric SAR," IEEE Trans. Geosci. Remote Sens. 35(1), 68-78 (1997).

14. Y. Boykov, O. Veksler, and R. Zabih, "Fast approximate energy minimization via graph cuts," IEEE Trans. Pattern Anal. Mach. Intell. 23(11), 1222-1239 (2001).

15. Y. Y. Boykov and M. P. Jolly, "Interactive graph cuts for optimal boundary and region segmentation of objects in n-d images," in Proc. Eighth IEEE Int. Conf. on Computer Vision (ICCV 2001), pp. 105-112 (2001).

16. S. S. Ganugapati and C. R. Moloney, "A ratio edge detector for speckled images based on maximum strength edge pruning," in Int. Conf. on Image Processing, pp. 2165-2165 (1995).

17. S. Deng et al., "Edge detection for polarimetric SAR images combining adaptive optimal polarimetric contrast enhancement and ROA operator," in IET Int. Radar Conf., pp. 1-6 (2013).

18. E. Zhang and Y. Zhang, F-Measure, pp. 1147-1147, Springer, New York (2009).

Xiaoqiang She received his BS degree from the University of Science and Technology of China (USTC), Hefei, China, in 2011, and his MS degree from the Institute of Electronics, Chinese Academy of Sciences (IECAS), Beijing, China, in 2014. He is currently working toward his $\mathrm{PhD}$ at the Key Laboratory of Technology, Geo-spatial Information Processing and Application System, IECAS. His current research interest is PolSAR image processing.

Xiaolan Qiu received her BS degree in electronic engineering and information science from the University of Science and Technology of China, Hefei, China, in 2004 and her PhD in signal and information processing from the University of Chinese Academy of Science, Beijing, China, in 2009. She is currently a vice professor of the Institute of Electronics, Chinese Academy of Sciences, Beijing, China. Her research interests include SAR image processing and SAR signal processing.

Bin Lei received his BS degree in Tsinghua University, Beijing, China, in 2000 and his $\mathrm{PhD}$ in signal and information processing from the University of Chinese Academy of Science, Beijing, China, in 2014. He is currently a professor of the Institute of Electronics, Chinese Academy of Sciences, Beijing, China. His research interests include SAR image processing and SAR signal processing. 